\title{
Autism-like Behaviors in Male Juvenile Offspring after Maternal Glyphosate Exposure
}

\author{
Yaoyu Pu${ }^{1}$, Li Ma ${ }^{1}$, Jiajing Shan ${ }^{1}$, Xiayun Wan ${ }^{1}$, Bruce D. Hammock ${ }^{2}$, Kenji Hashimoto ${ }^{1}$ \\ ${ }^{1}$ Division of Clinical Neuroscience, Chiba University Center for Forensic Mental Health, Chiba, Japan, ${ }^{2}$ Department of Entomology and \\ Nematology and UC Davis Comprehensive Cancer Center, University of California, Davis, CA, USA
}

\begin{abstract}
Objective: Exposure to the herbicide glyphosate during pregnancy and lactation may increase the risk for autism spectrum disorder (ASD) in offspring. Recently, we reported that maternal exposure of formulated glyphosate caused ASD-like behaviors in juvenile offspring. Here, we investigated whether maternal exposure of pure glyphosate could cause ASD-like behaviors in juvenile offspring.

Methods: Water or $0.098 \%$ glyphosate was administered as drinking water from E5 to P21 (weaning). Behavioral tests such as grooming test and three-chamber social interaction test in male offspring were performed from P28 to P35. Results: Male offspring showed ASD-like behavioral abnormalities (i.e., increasing grooming behavior and social interaction deficit) after maternal exposure of glyphosate.

Conclusion: The findings suggest that the exposure of glyphosate during pregnancy and lactation may cause ASD-like behavioral abnormalities in male juvenile offspring. It is likely that glyphosate itself, but not the other ingredients, may contribute to ASD-like behavioral abnormalities in juvenile offspring.
\end{abstract}

KEY WORDS: Autistic disorder; Ingredient; Glyphosate; Maternal exposure; Offspring.

\section{INTRODUCTION}

Autism spectrum disorder (ASD) is a complex neurodevelopmental disorder characterized by repetitive and characteristic patterns of behaviors and difficulties with social communications [1,2]. However, the detailed mechanisms underlying ASD etiology remain unknown. Growing evidence support a significant interaction of genetic and environmental factors in ASD etiology [1-4]. Environmental factors, including exposures to synthetic chemicals (i.e., herbicides) during pregnancy, may play a role in the etiology of ASD $[4,5]$.

Glyphosate [N-(phosphonomethyl)glycine], the active ingredient in Roundup ${ }^{\circledR}$ and other herbicides, is the most widely used herbicide in the world since it has excellent environmental profile and low toxicity $[6,7]$. A pop-

Received: September 14, 2020 / Accepted: September 25, 2020 Address for correspondence: Kenji Hashimoto

Division of Clinical Neuroscience, Chiba University Center for Forensic Mental Health, 1-8-1 Inohana, Chuo-ku, Chiba 260-8670, Japan

E-mail: hashimoto@faculty.chiba-u.jp

ORCID: https://orcid.org/0000-0002-8892-0439 ulation-based study in California of the United State (US) demonstrated that the risk of ASD might be associated with exposure of glyphosate during pregnancy and early childhood [8], suggesting a possible relationship between maternal glyphosate exposure and ASD in offspring $[9,10]$. A recent systemic review shows that glyphosate showed a "moderate level of evidence" in their association with ASD in children [11]. In a rodent study, we recently reported that maternal exposure to high dose of formulated glyphosate (Roundup ${ }^{\circledR}$ Maxload) caused ASD-like behavioral abnormalities in male juvenile offspring [12]. The surfactant, polyethoxylated tallow amine, contained in the formulated glyphosate such as Roundup [13]. However, how glyphosate or other ingredients in the Roundup ${ }^{\circledR}$ Maxload contribute to ASD-like behaviors in offspring is unknown.

Here, we investigated whether maternal exposure of pure glyphosate causes ASD-like behaviors in male juvenile offspring.

(c) This is an Open-Access article distributed under the terms of the Creative Commons Attribution Non-Commercial License (http://creativecommons.org/licenses/by-nc/4.0) which permits unrestricted non-commercial use, distribution, and reproduction in any medium, provided the original work is properly cited. 


\section{METHODS}

\section{Animals}

Pregnant ddY mice (embryo at the 5th day [E5], 9-10 weeks old) were purchased from Japan SLC Inc. (Shizuoka, Japan). Each pregnant mouse was housed singly in clear polycarbonate cage $(22.5 \times 33.8 \times 14.0 \mathrm{~cm})$ under controlled temperatures and 12 hour light/dark cycles (lights on between 07:00 - 19:00 h), with ad libitum food (CE-2; CLEA Japan, Inc., Tokyo, Japan) and water. This study was approved by the Chiba University Institutional Animal Care and Use Committee (permission number: 30-352 and 1-135).

\section{Treatment of Glyphosate in Drinking Water into Pregnant Mice}

The commercially available glyphosate (Lot number: MKCK2795; Sigma-Aldrich Co., Ltd., Tokyo, Japan) was used. Previously, we used $0.098 \%$ formulated glyphosate (expressed as $0.025 \%$ Roundup ${ }^{\circledR}$ Maxload [48\% (w/v) glyphosate potassium salt, $52 \%$ other ingredients such as water and surfactant])[12,14]. Therefore, pregnant mice were treated with water or $0.098 \%$ pure glyphosate from E5 to P21 (weaning) (Fig. 1A). The male offspring were isolated from their mothers at weaning (P21), and the mice were caged each three-five in the groups in clear polycarbonate cage $(22.5 \times 33.8 \times 14.0 \mathrm{~cm})$. Mice were housed under controlled temperatures and 12 hour light/dark cycles (lights on between 07:00 - 19:00 h), with ad libitum food and water.

\section{Behavioral Analysis}

Behavioral tests of male offspring were performed from P28 to P35 (Fig. 1A). The grooming test was performed as previously described [12]. Each mouse was put in a clean standard mouse cage separately and allowed to adjust for 10 minutes. A video camera (C920r HD Pro; Logitech International SA, Tokyo, Japan) was fixed two meters in front of the cage to record the mice behavior for the next 10 minutes, following the habituation time. After the experiment, the total self-grooming time of each subject mouse was counted by an experimenter through watching these videos. A timer was prepared for scoring cumulative time spent grooming during the 10 minutes test session.

The three-chamber social interaction test was performed as reported previously [12]. The testing apparatus consisted of a clear rectangular box and a lid with a video camera. Two clear plastic dividers with a small square opening $(5 \times 8 \mathrm{~cm})$ divided the box into three equal chambers $(20 \times 40 \times 20 \mathrm{~cm})$. Also, an empty wire cage $(10 \mathrm{~cm}$ in diameter, $17.5 \mathrm{~cm}$ in height, with vertical bars $0.3 \mathrm{~cm}$ apart) was put in the center of each left and right chamber. First, each test mouse was placed in the chamber and allowed to explore the entire box for 10 minutes to habituate the environment. Next, a ddY male mouse (stranger 1) that never meet the test mouse before was put into a wire cage that was located into one of the side chambers. To evaluate sociability, the test mouse was allowed to explore the box for an additional 10 minutes. Last, to assess social novelty, a second stranger male mouse (stranger 2)
A

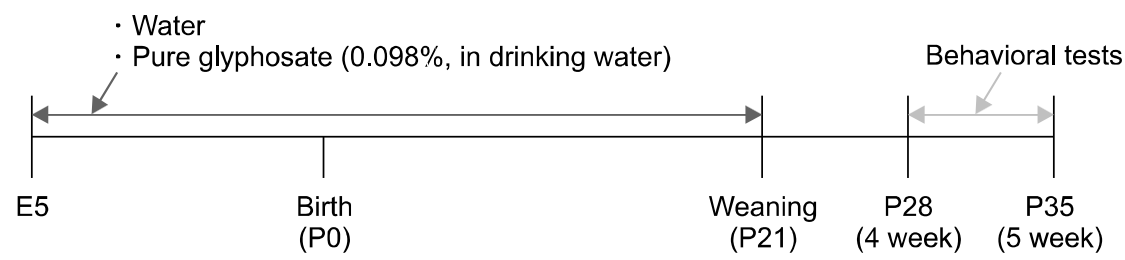

B

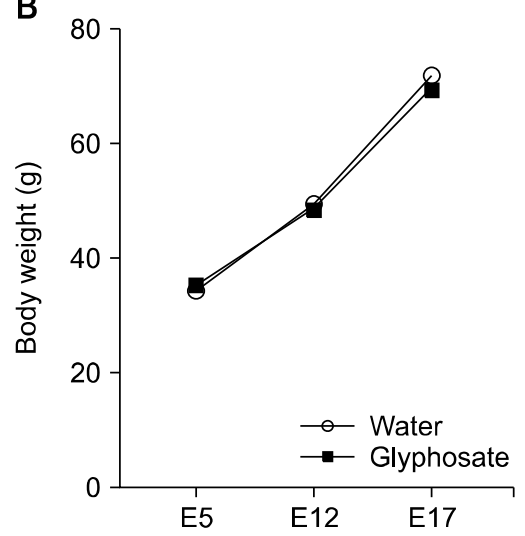

Fig. 1. Treatment schedule and body weight changes of mothers. (A) Schedule of treatment, and behavioral tests of offspring. (B) Change of body weight of pregnant mothers. Repeated measure one-way ANOVA $\left(\mathrm{F}_{1,9}=0.246, p=0.632\right)$. Data are shown as mean \pm standard error of the mean $(n=5$ or 6$)$. 

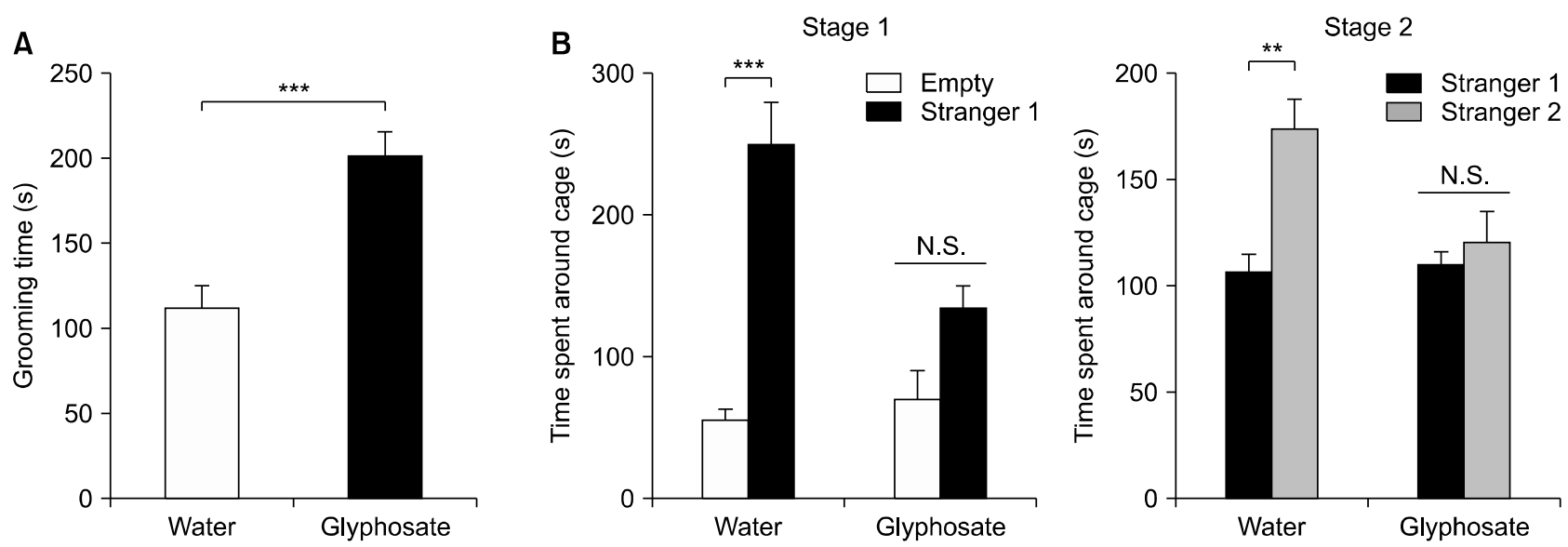

Fig. 2. Autism spectrum disorder-like behavioral abnormalities in male juvenile offspring. (A) Grooming test. Male juvenile offspring after maternal glyphosate exposure showed the increased grooming time compared to control group. Data are shown as mean \pm standard error of the mean (SEM) $(\mathrm{n}=8) .{ }^{* * *} p<0.01$ compared to control group (Student $\mathrm{t}$ test). (B) Three chamber social interaction test. Left: Two-way ANOVA (glyphosate: $\mathrm{F}_{1,28}=$ 6.929, $p=0.014$; stranger: $\mathrm{F}_{1,28}=46.21, p<0.001$; interaction: $\mathrm{F}_{1,28}=11.89, p=0.002$ ). Right: Two-way ANOVA (glyphosate: $\mathrm{F}_{1,28}=5.098, p=$ 0.032 ; stranger: $\mathrm{F}_{1,28}=12.36, p=0.002$; interaction: $\left.\mathrm{F}_{1,28}=6.609, p=0.016\right)$. Data are shown as mean $\pm \operatorname{SEM}(\mathrm{n}=8) .{ }^{* *} p<0.01,{ }^{* * *} p<0.001$ compared to control group.

N.S., not significant.

was placed into the wire cage that had been empty during the first 10 minutes, and the test mouse was allowed another 10 minutes to interact with the strangers and to explore the box. Thus, the subject mouse had a choice between the first, non-familiar mouse (stranger 1 ) and the novel unfamiliar mouse (stranger 2). The time spent in each chamber and the interaction time spent around each cage was recorded on video.

\section{Statistical Analysis}

Analysis of the data was performed using GraphPad Prism ver. 7 (GraphPad Software, La Jolla, CA, USA). The data of body weight changes were analyzed using the repeated measure one-way analysis of variance (ANOVA), followed by post-hoc Bonferroni test. Comparisons between two groups were performed using Student $t$ test. The data of three-chamber social interaction test were analyzed using two-way ANOVA, followed by post-hoc Bonferroni test. The $p$ values of less than 0.05 was considered statistically significant.

\section{RESULTS}

\section{Behavioral Abnormalities in Juvenile Offspring after Maternal Glyphosate Exposure}

There were no changes in body weight of glyphosate-treated mothers and water-treated mothers (Fig. 1B).
In the grooming test, male juvenile offspring after maternal glyphosate exposure showed increased grooming time compared to the water-treated group (Fig. 2A). In the three-chamber social interaction test, male juvenile offspring after maternal glyphosate exposure showed social interaction deficits compared to the water-treated group (Fig. 2B). The data suggest that the exposure to pure glyphosate during pregnancy and lactation causes ASD-like behavioral abnormalities in male juvenile offspring.

\section{DISCUSSION}

In this study, we found that the exposure to high levels $(0.098 \%)$ glyphosate during pregnancy and lactation caused ASD-like behaviors in male juvenile offspring. These findings are consistent with previous report [12] using formulated glyphosate $(0.098 \%)$. Previously, we used the commercially available Roundup ${ }^{\circledR}$ Maxload, although the detailed information of surfactant was not disclosed. The current data using pure glyphosate (Sigma-Aldrich Co., Ltd., Tokyo, Japan) are similar to the results of the previous report using Roundup ${ }^{\mathbb{R}}$ Maxload [12]. Collectively, it is likely that glyphosate itself, but not ingredient, might contribute to ASD-like behavioral abnormalities in offspring after maternal exposure.

In this study, we did not find the body weight changes between two groups, inconsistent with the previous data 
using formulated glyphosate [12]. Thus, it is likely that the ingredients in the Roundup ${ }^{\circledR}$ Maxload contribute to decreased body weight of pregnant mice after formulated glyphosate exposure. In addition, the reduction of body weight of pregnant mice might not contribute to ASD-like behaviors in male offspring after maternal glyphosate exposure.

Maternal exposure to $0.098 \%$ glyphosate might cause ASD-like behaviors in juvenile offspring, although it is exceptionally unlikely that such exposure could be reached during human pregnancy $[12,15]$. A prospective birth cohort study in Central Indiana of US demonstrated that $>$ $90 \%$ of pregnant women had detectable levels of glyphosate $(0.1 \mu \mathrm{g} / \mathrm{L})$ in the urine [16]. Furthermore, higher urine levels of glyphosate were significantly correlated with shortened pregnancy lengths $(r=-0.28, p=0.02)$ [16]. A recent review shows the detection of urine levels of glyphosate in individuals exposed occupationally, para-occupationally, or environmentally to the herbicide [17]. Therefore, it is of great interest to investigate a cohort study on measurement of blood (or urine) levels of glyphosate and its metabolite aminomethylphosphonic acid in pregnant mothers who have their offspring with or without ASD.

This paper has limitation. In this study, we used male offspring after maternal glyphosate exposure since the prevalence of ASD in male subjects is higher than that in female subjects [18]. Nonetheless, it is also of interest to examine whether maternal glyphosate exposure could cause ASD-like phenotypes in female offspring.

In conclusion, this study suggests that the exposure to pure glyphosate during pregnancy and lactation might play a role in the etiology of ASD-like behaviors in male juvenile offspring. Therefore, it is likely that glyphosate itself, but not the other ingredients, may contribute to ASD-like behavioral abnormalities in male juvenile offspring although further detailed study is needed.

\section{Acknowledgments}

This study was supported by the Japan Society for the Promotion of Science (JSPS) KAKENHI (to K.H., 17H04243), the National Institute of Environmental Health Sciences (NIEHS) River Award R35 ES030443-01 (to B.D.H.), NIEHS Superfund Program P42 ES004699 (to B.D.H.).

\section{- Conflicts of Interest}

No potential conflict of interest relevant to this article was reported.

\section{Author Contributions}

Conceptualization: Yaoyu Pu, Bruce D. Hammock, Kenji Hashimoto. Data acquisition: Yaoyu Pu, Li Ma, Jiajing Shan, Xiayuan Wan. Formal analysis: Yaoyu Pu, Li Ma, Jiajing Shan, Xiayuan Wan. Supervision: Kenji Hashimoto. Writing-original draft: Yaoyu Pu, Kenji Hashimoto. Writing - review \& editing: Yaoyu Pu, Bruce D. Hammock, Kenji Hashimoto.

\section{ORCID}

Yaoyu Pu https://orcid.org/0000-0002-7887-3237

Li Ma https://orcid.org/0000-0002-7157-7144 Jiajing Shan https://orcid.org/0000-0003-2875-1941 Xiayun Wan https://orcid.org/0000-0002-5173-2071

Bruce D. Hammock https://orcid.org/0000-0003-1408-8317 Kenji Hashimoto https://orcid.org/0000-0002-8892-0439

\section{REFERENCES}

1. Lai MC, Lombardo MV, Baron-Cohen S. Autism. Lancet 2014; 383:896-910.

2. Lord C, Elsabbagh M, Baird G, Veenstra-Vanderweele J. Autism spectrum disorder. Lancet 2018;392:508-520.

3. Hallmayer J, Cleveland S, Torres A, Phillips J, Cohen B, Torigoe T, et al. Genetic heritability and shared environmental factors among twin pairs with autism. Arch Gen Psychiatry 2011;68:1095-1102.

4. Sealey LA, Hughes BW, Sriskanda AN, Guest JR, Gibson AD, Johnson-Williams L, et al. Environmental factors in the development of autism spectrum disorders. Environ Int 2016;88: 288-298.

5. Kim JY, Son MJ, Son CY, Radua J, Eisenhut M, Gressier F, et al. Environmental risk factors and biomarkers for autism spectrum disorder: an umbrella review of the evidence. Lancet Psychiatry 2019;6:590-600.

6. Bradberry SM, Proudfoot AT, Vale JA. Glyphosate poisoning. Toxicol Rev 2004;23:159-167.

7. Kier LD, Kirkland DJ. Review of genotoxicity studies of glyphosate and glyphosate-based formulations. Crit Rev Toxicol 2013;43:283-315.

8. von Ehrenstein OS, Ling C, Cui X, Cockburn M, Park AS, Yu F, et al. Prenatal and infant exposure to ambient pesticides and autism spectrum disorder in children: population based casecontrol study. BMJ 2019;364:1962.

9. Bakian AV, VanDerslice JA. Pesticides and autism. BMJ 2019; 364:/1149.

10. Rueda-Ruzafa L, Cruz F, Roman P, Cardona D. Gut microbiota and neurological effects of glyphosate. Neurotoxicology 2019;75:1-8. 
11. Ongono JS, Béranger R, Baghdadli A, Mortamais M. Pesticides used in Europe and autism spectrum disorder risk: can novel exposure hypotheses be formulated beyond organophosphates, organochlorines, pyrethroids and carbamates? - a systematic review. Environ Res 2020;187:109646.

12. Pu Y, Yang J, Chang L, Qu Y, Wang S, Zhang K, et al. Maternal glyphosate exposure causes autism-like behaviors in offspring through increased expression of soluble epoxide hydrolase. Proc Natl Acad Sci U S A 2020;117:11753-11759.

13. Meftaul IM, Venkateswarlu K, Dharmarajan R, Annamalai P, Asaduzzaman M, Parven A, et al. Controversies over human health and ecological impacts of glyphosate: is it to be banned in modern agriculture? Environ Pollut 2020;263(Pt A): 114372.

14. Pu Y, Chang L, Qu Y, Wang S, Tan Y, Wang X, et al. Glyphosate exposure exacerbates the dopaminergic neurotoxicity in the mouse brain after repeated administration of MPTP. NeurosCi Lett 2020;730:135032.

15. Solomon KR. Estimated exposure to glyphosate in humans via environmental, occupational, and dietary pathways: an updated review of the scientific literature. Pest Manag Sci 2019; 76:2878-2885.

16. Parvez S, Gerona RR, Proctor C, Friesen M, Ashby JL, Reiter JL, et al. Glyphosate exposure in pregnancy and shortened gestational length: a prospective Indiana birth cohort study. Environ Health 2018; 17:23.

17. Gillezeau C, van Gerwen M, Shaffer RM, Rana I, Zhang L, Sheppard L, et al. The evidence of human exposure to glyphosate: a review. Environ Health 2019;18:2.

18. Werling DM, Geschwind DH. Sex differences in autism spectrum disorders. Curr Opin Neurol 2013;26:146-153. 